\title{
Donner du sens aux savoirs: la didactique, quarante ans après ${ }^{1}$
}

\section{Dando sentido aos saberes: a didática, quarenta anos depois}

\section{Providing meanings to knowledge: didactics, forty years later}

\author{
Michel Develay²
}

\begin{abstract}
RESUME
La question centrale posée dans de nombreux pays concerne le sens des savoirs enseignés, interrogation à laquelle la didactique souhaite apporter sa contribution. Le texte joint s'intéresse à cette discipline à travers trois focales: a) l'émergence du regard didactique en France, il y a une quarantaine d'années, ses causes et la manière dont la didactique a pris ses distances d'avec la pédagogie; b) l'évolution de la réflexion didactique créatrice de concepts, jusqu'à son état actuel relativement amorphe; c) la didactique aujourd'hui, affrontant trois interrogations au cœur de l'école: la question du sens, l'énigme du rapport au savoir et la place de la Culture.

Mots-clés: savoirs enseignés; didactique en France; Culture.
\end{abstract}

\section{RESUMO}

Em vários países a questão central se dá em torno dos saberes ensinados, interrogação que a didática deseja trazer sua contribuição. Este texto se interessa por essa disciplina através de três focos: a) a emergência do olhar didático na França há quarenta anos, suas causas e a maneira que a didática se distanciou da pedagogia; b) a evolução da reflexão didática criadora de

DOI: $10.1590 / 0104-4060.43363$

1 Conferência proferida no $9^{\circ}$ Colóquio EDUCON - Educação e Contemporaneidade na Universidade Federal do Sergipe, Aracaju em setembro/2015.

2 Université Lumière Lyon 2. Lyon, França.86 Rue Pasteur, 69007.E-mail: md.develay@ orange.fr 
conceitos, até seu estado atual relativamente amorfo; c) a didática hoje, enfrentando três interrogações no coração da escola: a questão do sentido, o enígma da relação com o saber e o lugar da Cultura.

Palavras-chave: saberes ensinados; didática na França; Cultura.

\begin{abstract}
In many countries the central subject is about the taught knowledge, a question that didactics aims to bring as its contribution. This text presents interest for that discipline through three points: a) the emergence of a didactic view in France for forty years, its causes and the way didactics became distant from pedagogy; b) the didactic reflection evolution which created concepts, until its current situation that is kind of amorphous; c) didactics today, facing three questions in the heart of schools: the subject of meaning, the puzzle of relating knowledge and the place of Culture.
\end{abstract}

Keywords: taught knowledge; didactics in France; Culture.

Dans de nombreux pays les didactiques des disciplines (D des D) constituent une matière académique présente au sein des universités et/ou des centres de formation d'enseignants. Les D. des D. se centrent sur les processus d'enseignement, d'apprentissage et de formation non pas en général, mais au sein d'une discipline donnée. Elles font ainsi l'hypothèse que la nature d'une discipline détermine les conditions de réussite des apprentissages ou de la formation qui lui sont liées. Ainsi le didacticien des mathématiques conjecture que si un élève est plus à l'aise dans l'apprentissage de cette discipline que dans l'apprentissage de sa langue maternelle, c'est parce qu'il entretient un rapport de plus grande proximité avec les maths qu'avec sa langue maternelle.

Historiquement, l'émergence des didactiques des disciplines est relativement récente: moins d'une quarantaine d'années en France.

Cet exposé vise d'abord à décrire l'évolution des D. des D., en prenant comme point d'appui la didactique des sciences. Ensuite il cherchera à problématiser les rapports entre les D. des D. et trois questions actuelles qui traversent l'institution scolaire en France:

- la question du sens de l'école et des savoirs. Baignés dans une société qui elle-même cherche un sens à son devenir, affrontés à une incertitude quant au lien réussite scolaire/ réussite sociale, confrontés aux nouvelles technologies, les élèves se demandent de plus en plus fréquemment quel sens conserve l'école et les savoirs qui y sont enseignés, ces derniers étant en libre accès sur les écrans, la réussite scolaire n'étant pas toujours un gage de réussite sociale; 
- la question du rapport au savoir ensuite. Le regard didactique préjuge que les questions d'apprentissage sont en rapport étroit avec les contenus enseignés. Dès lors, les didactiques des disciplines se distinguent-elles de cette notion dont Bernard Charlot a été un des initiateurs clés;

- la question de la Culture enfin qui constitue un des fondements de l'école et qui ressurgit dans le contexte français. Comment les D. des D. peuvent-elles faciliter les apprentissages scolaires et être attentives à une école lieu d'accès à la Culture?

\section{Le projet initial des $D$. des D. en France}

En France, la D. des D. est apparue presque simultanément en mathématiques et en sciences dans les années 1970-1975. Il y a quarante ans.

En mathématiques, la $\mathrm{D}$. des $\mathrm{D}$ a découlé d'universitaires confrontés à une double interrogation:

- Une première interrogation était relative à la nature même des mathématiques. A la suite du traumatisme pour les Etats-Unis que constitua l'envoi du Spoutnik russe sur la lune, la vision des mathématiques institué dans ce pays donna forme aux News maths traduites en français par les mathématiques modernes. Il s'agissait d'une toute autre approche des mathématiques, imprégnées par le structuralisme. La théorie des ensembles reconsidérait par exemple totalement l'arithmétique.

- Une seconde interrogation prenait forme à propos de l'élève. Les événements de 1968 conduisaient à le considérer davantage comme acteur et non plus sujet de ses apprentissages. Jean Piaget constitua la référence en matière de pédopsychologie cognitive. La $\mathrm{D}$. des mathématiques conduira ainsi à faire travailler ensemble des mathématiciens, des psychologues, des sociologues et des linguistes. Ce champ de recherches s'institutionnalisera avec la création au sein des universités d'Instituts de recherche sur l'enseignement des mathématiques (les IREM créés en 1968), puis l'existence d'enseignements doctoraux (les premiers en 1975), d'un séminaire national (1978), et d'une revue nationale en $1980^{3}$.

$3<$ http://www.persee.fr/web/revues/home/prescript/article/rfp_0556-7807_1986 num_76_1_1503>. Accès le: 22 fév. 2015. 
L'émergence d'une didactique des sciences (regroupant physique, technologie et biologie) est concomitante à la $\mathrm{D}$. des mathématiques et elle suivra un cheminement parallèle mais non identique.

- Parallèle car il résultera d'un questionnement à propos des contenus et du statut de l'élève. Ainsi en biologie, l'émergence de la biologie moléculaire avec les trois Nobel français Jacob, Monod et Lwoff en 1965 posera la question du quoi enseigner et fera émerger un nouveau paradigme pour expliquer le vivant à partir de la cellule et non pas de l'organisme. Ici encore les événements de 1968 développeront une plus vigilance sur la prise en compte de la pensée de l'élève et Jean Piaget constituera une référence scientifique forte. Ce cheminement se traduira ici encore par des enseignements doctoraux, des revues, des séminaires.

- Mais non identique car les concepts travaillés, donnant corps à la D. des sciences ne seront pas les mêmes qu'en D. des mathématiques. Nous y reviendrons dans un instant.

L'installation des D. des D. comme domaines de recherche dans le domaine universitaire sera amplifiée par l'existence à partir de 1980 de nouveaux centres de formation des enseignants (les IUFM) comme substitute des Ecoles normales. Pour les formateurs de ces centres, la didactique constituera la figure tutélaire à laquelle se raccrocher. A l'origine professeurs d'une discipline, les enseignants en IUFM seront nommés formateurs, et enfin didacticiens.

Par la suite, chacune à son rythme verront le jour une D. du français, une $\mathrm{D}$. des arts plastiques, une $\mathrm{D}$. des langues étrangères, une $\mathrm{D}$. des sciences humaines, etc.

Par la soutenance de thèses, la création de postes universitaires, l'existence de revues, de colloques, de séminaires, depuis 1990-1995, beaucoup a été formalisé et institutionnalisé pour qu'en France les D. des D. constituent un domaine de recherche, d'action et de formation bien identifié. Nous n'envisagerons pas les reconfigurations auxquelles cette entreprise a donné lieu au plan spéculatif et au plan institutionnel. Ainsi, certains pédagogues s'en détourneront considérant que la pédagogie s'était toujours intéressée aux contenus, certains philosophes tout autant, dénonçant l'absence de prise en compte de la question des valeurs ou d'une éthique de la communication, certains sociologues s'y intéresseront indirectement avec la sociologie du curriculum. 


\section{L'évolution de la réflexion concernant la $\mathrm{D}$. des disciplines}

Nous avons parlé jusqu'alors de l'émergence de la D. des disciplines liée à trois phénomènes: une interrogation sur les contenus à enseigner, un nouveau regard porté sur l'élève et enfin des interrogations à propos de la formation.

Arrêtons-nous maintenant sur l'évolution que cette D. des disciplines a connue dans les quarante dernières années.

a) On peut lire cette évolution au niveau des concepts mobilisés par les différentes didactiques depuis 1970.

Une étude discipline académique par discipline académique reste à conduire. On découvrirait comment la $\mathrm{D}$. de chacune de ces disciplines académiques a particularisé certains concepts.

Ainsi en mathématiques les deux premiers concepts mobilisés ont été ceux de transposition didactique (comment un savoir académique ou une pratique sociale existant en dehors du système scolaire sont-ils réinterprétés aux différents niveaux d'enseignement) et de contrat didactique (une discipline enseignée conduit-elle à un type particulier de relation entre maître et élèves et entre élèves entre eux). Transposition didactique, et donc attention forte aux contenus peut-être parce que les savoirs en mathématiques ne sont que peu affectés par des pratiques sociales comme c'est le cas en sciences, mais aussi parce que les universitaires étaient à la pointe de la didactique naissante. Contrat didactique car le champ d'intersubjectivités formels et non formels sauta aux yeux d'universitaires découvrant la réalité des classes.

En sciences ce furent les concepts de pratique sociale, de représentation, et d'objectif-obstacle qui émergèrent en priorité. Représentation et objectif-obstacle davantage que transposition peut-être parce que les initiateurs de la D. des sciences étaient davantage des enseignants dans les centres de formation innovateurs que des universitaires comme en mathématiques.

b) On peut lire cette évolution au niveau des concepts nouveaux créés par les différentes didactiques.

Pierre Thuillier, un historien des sciences, dans "Jeux et enjeux de la science" explique, en s'appuyant sur l'exemple de la biologie moléculaire, et sur la notion de paradigme chez Kuhn, que la vie d'une science peut se décliner en trois étapes.

- D'abord existerait une phase romantique. Elle donnerait lieu à un foisonnement de recherches, d'idées nouvelles, correspondant à une 
nouvelle orientation du regard, à l'émergence d'un nouveau paradigme. C'est le moment où des nouvelles questions sont posées, où des nouvelles solutions apparaissent. On ne regarderait plus les choses comme précédemment. C'est ce que permirent en $\mathrm{D}$. des maths et des sciences les notions de transposition didactique ou de représentation.

- Succéderait une phase dynamique, occasion d'apporter des réponses nouvelles à des problèmes jusqu'alors non résolus à partir des concepts créés en nombre lors de la phase romantique. A partir de la notion de transposition didactique on se met à regarder différemment les contenus enseignés à différents niveaux de classe pour rechercher quelle complexité les affecte, le lien le cas échéant que ces notions entretiennent avec des pratiques sociales. Avec la notion de représentation, on se met à considérer que tout apprenant mais aussi tout enseignant véhicule ses propres représentations vis-à-vis du contenu enseigné ou à acquérir, et qu'il existe une différence entre un contenu acquis et un contenu approprié.

- Une phase académique succédant à la phase romantique conduirait à la stabilisation du cadre de recherche, jusqu'à ce qu'une révolution scientifique vienne reconsidérer ce paradigme et en suggérer un autre. Cette dernière phase montrerait en quelque sorte la limite de la science. Et je pense qu'on peut se demander si les D. des D. ne sont pas actuellement dans une phase académique, car elles ne créent plus de concepts nouveaux.

La conclusion de ce trop rapide tour d'horizon? Je postule que la D. est aujourd'hui à un point mort: elle utilise ses questionnements et ses concepts sur les différents contenus de savoirs de la discipline à laquelle elle s'intéresse.

Aussi les didacticiens ont à se demander comment s'intéresser aux nouveaux questionnements qui émergent des élèves dans une école qui massifie son accès sans forcément le démocratiser. Et, je l'ai suggéré en introduction: des questionnements nouveaux apparaissent au sein de l'école. Ces questionnements confluent autour de la question du sens, du rapport au savoir et de la culture.

J'ai donc intitulé cette troisième partie: le Didactique aujourd'hui à la croisée de trois questionnements posés à toute les écoles: la question du sens, de la culture et du rapport au savoir. 


\section{Le Didactique aujourd'hui: à la croisée de trois questionnements: la question du sens, de la culture et du rapport au savoir}

\section{a) La didactique et le sens.}

Cette conférence n'a aucun sens. Le sens ne gît pas dans une situation. En soi l'EPS n'a pas plus ou moins de sens que les mathématiques. Vous trouverez du sens à mon intervention ou vous n'en trouverez pas. Le sens est personnel, rarement définitif et pour reprendre Gilles Deleuze dans son ouvrage "Les logiques du sens", le sens est dans le rapport de soi avec soi, de soi avec les autres, de soi avec le monde.

Vous trouverez du sens dans mon propos peut-être parce qu'il répond à une question que vous vous êtes posée et qui n'avait pas de réponse ou parce qu'il pose une question que vous ne vous étiez pas posée. Le sens est dans le rapport de soi avec soi.

Ou bien cette conférence aura du sens parce qu'elle vous conduit à reconsidérer votre manière de penser ou d'agir avec vos élèves, ou avec vos collègues ou avec votre administration à travers le curriculum qui préexiste, les activités ou les évaluations que vous conduisez. Le sens est alors dans le rapport de soi avec les autres. Du reste vous trouverez du sens à ce colloque parce qu'il vous aura permis d'échanger avec un collègue que vous ne connaissiez pas.

Ou bien cette conférence aura du sens parce qu'elle vous ouvre à des réflexions qui débordent votre personne, votre situation professionnelle et qui débouchent sur des pensées mettant en jeu le statut de l'humaine humanité. Le sens est dans le rapport de soi avec le monde. Entendant mon intervention de français, vous pourrez peut-être lui trouver du sens parce qu'elle voisine des questionnements brésiliens.

Dans un livre qui a maintenant une vingtaine d'années "Donner du sens à l'école", je suggérais pour aider un élève à trouver du sens à l'école, quatre manières de faire en le considérant:

- comme élève épistémologue. En le rendant apte à se mettre à distance des savoirs à maîtriser en les situant au sein de la discipline enseignée, en appréciant les relations avec d'autres savoirs de la même discipline ou d'autres disciplines, en se familiarisant avec les conditions historiques et sociales de leur émergence;

- comme élève stratège. Il a conscience de ce à quoi peuvent lui être utiles les savoirs acquis, tant au niveau d'un projet personnel, qu'au niveau d'un projet professionnel. Il est capable de faire des choix dans ses investissements scolaires; 
- comme élève méthodologue. Il a acquis un recul suffisant par rapport à la manière de se comporter face à un enseignant ou face à une tâche pour procéder de la meilleure manière possible. Il s'est donné une méthode;

- comme élève analysant. Il est à même d'expliquer comment il a acquis tel ou tel savoir, comment il est possible de développer de la réflexivité par le jeu de la métacognition.

Les D. des disciplines se sont tournées vers la question du sens en mettant l'accent au plan pédagogique sur la notion de problématisation et d'inquiry, en s'intéressant aux situations-problèmes, aux représentations des élèves et au-delà à l'épistémologie et donc à la structure des savoirs à l'histoire des savoirs et donc à l'idée d'obstacle épistémologique.

Je suggère que les $\mathrm{D}$. des disciplines théorisent davantage la question du sens au sein de leur matière. Peut-être en arriveront-elles à considérer avec le sociologue Alain Touraine que le sens est dans l'ambition démocratique attentive de l'accès à la raison, à la valeur de l'histoire et à la recherche de la liberté. Aider les élèves à trouver du sens dans un enseignement de biologie conduirait à emprunter à l'histoire des sciences pour montrer le caractère tout à la fois reconnu et incertain des notions, à illustrer l'idée d'obstacle épistémologique, de réfutation... Alors aider les élèves à trouver du sens dans un enseignement de biologie conduirait à leur faire découvrir le rapport entre les savoirs en jeu et certains débats démocratiques (des questionnements à propos de notre alimentation, du devenir de la planète, de la procréation ou de la fin de vie, du lien entre biologie et médicamentation, entre productions végétales ou animales et circuits courts pour l'alimentation...).

\section{b) Le rapport au savoir et la $D$.}

Parler de rapport au savoir c'est convenir que les élèves entretiennent une certaine liaison, un certain commerce avec le savoir. On parle de rapport au savoir comme on parle de rapport amoureux. Le rapprochement entre rapport au savoir et rapport amoureux n'est du reste pas totalement fortuit. Freud n'a t-il pas écrit que "apprendre, c'est investir du désir dans un objet de savoir"? Il n'y a pas d'apprentissage scolaire sans désir d'apprendre, sans chercher à vivre avec le savoir et ce que représente son acquisition, une liaison de plaisir, une liaison de nature érotique.

Cependant plutôt qu'employer les idées de liaison, de relation, ou de commerce avec le savoir, nous préférons l'expression de "rapport au savoir". Le terme "rapport à..." est plus énigmatique que les précédents. Le rapport d'un sujet au savoir induit que quelque chose de lâche, de non prémédité, de flottant 
existe entre lui et le savoir. L'idée de "rapport à..." renvoie à un processus vraisemblablement non conscient, non prémédité, non voulu entre une personne et un savoir. B. Charlot considère d'une part, que ce qui s'exprime dans le rapport au savoir c'est l'identité même de l'individu constituée par une "constellation de repères, de pratiques, de mobiles et de buts engagés dans le temps", et d'autre part, qu'il est pertinent et légitime de parler de rapport au savoir d'un groupe car le rapport au savoir d'une personne émerge du rapport au savoir du (ou des) groupes auxquels il appartient (sa famille, son milieu social).

Les D. se sont constituées en préjugeant l'importance de la spécificité d'un contenu pour son appropriation. Les D. font ainsi indirectement le pari que le rapport au savoir de l'élève est déterminant pour son appropriation. Jusqu'alors l'épistémologie répondait à cette situation. C'est en elle que résidait la spécificité d'un contenu à enseigner. Dit autrement, c'est la nature des questions auxquelles répond une discipline, c'est la spécificité de la méthode pour répondre à ces questions et c'est l'ensemble des faits, notions, théories et le paradigme de cette discipline qui instituait un rapport de proximité ou de distance de l'élève avec cette discipline.

Les D. des disciplines tangentent donc la question du rapport au savoir mais dans un registre cognitif. Il serait intéressant, reprenant certaines notions comme par exemple celles de représentations sociales de prolonger cette réflexion dans une approche davantage sociologique et simultanément psychanalytique tant le rapport au savoir n'est pas dans un rapport de superficialité mais de profondeur, dans un rapport identitaire.

\section{c) La culture, l'ontologie et la D.}

Le projet scolaire peut être énoncé de plusieurs manières.

A la question "pour quoi l'école?", Emile Durkheim, le père de la sociologie, répond: pour former le travailleur, pour former le citoyen et pour former la personne. Le projet de l'école n'est pas de former la personne ou le travailleur, mais de s'intéresser aux deux simultanément, ce qui n'est pas évident par les temps qui courent.

A la question “pourquoi l'école?", il est aussi possible de répondre: la socialisation par l'éducation. En ne privilégiant donc pas d'un côté la classe et les savoirs qui y sont dispensés et de l'autre côté la cour ou les activités périscolaires qui permettraient d'intérioriser les normes et les valeurs de la société à laquelle il appartient, construisant alors son identité sociale. La finalité de l'acquisition des savoirs est au delà des savoirs, elle est au service de la socialisation ${ }^{4}$.

4 On pourrait aussi énoncer que le projet scolaire est de faire émerger de l'altérité dans une relation de parité asymétrique. Altérité car l'élève sera différent de ce que souhaite en faire l'ensei- 
Cette dernière convention fait toute sa place à l'idée de culture comme finalité ultime de l'école. Socialiser par les savoirs conduit à placer les savoirs au service de la culture. Ainsi la culture est davantage que la somme des savoirs et donc que la somme des disciplines. Et je pense en effet que la culture que l'école vise à faire s'approprier aux élèves est constituée, à travers chaque discipline enseignée de la réponse à des questions de nature ontologique. Je m'explique.

En arithmétique, avec le nombre, l'homme approche l'infini et l'incommensurable. L'infini, par l'infiniment grand car il n'y a pas débuté à la numération (les nombres peuvent aller jusqu'au ciel disent les jeunes enfants), et par l'infiniment petit car entre deux nombres décimaux qui se succèdent existe une infinité de décimaux. L'incommensurable, car $22 / 7^{\text {ème5 }}$ qui est $\pi$ est bien un nombre mais incommensurable car il est constitué d'un infini de chiffres après la virgule. L'infini des mathématiques qui peut être rapporté au temps et à l'espace embraie sur l'infini théologique. Pour le croyant, Dieu est considéré comme le marqueur de l'infini par ses deux attributs que sont l'éternité et l'immensité. Dieu ne pouvant être limité, c'est sa perfection même qui est infinie. De par son infinité, Dieu est d'ailleurs le terme ultime de la série infinie des faits contingents du monde, en tant que raison suffisante dernière. La numération est une occasion d'établir un pont avec des questions métaphysiques. Pour le non croyant, l'infini est tout autant présent dans l'infinité de questionnement lié à une vision matérialiste du hasard et de la nécessité à l'origine de l'homme.

Avec la géométrie l'élève se confronte à la vision et à la compréhension d'objets réels ou idéalisés dans l'espace. La droite dont il est si difficile de définir la nature (un ensemble de points?), est impossible de dessiner (elle est infinie et devrait donc déborder de la feuille sur laquelle on la trace), tout comme le cercle ou n'importe quelle figure géométrique ne sont que des idéaux pour la pensée. Il ne sera jamais possible de dessiner un cercle qui dans son idéalité désigne l'ensemble des points situés à égale distance d'un point fixe, le centre. La géométrie nous confronte à la question du réel, en montrant qu'il est inaccessible.

Avec les probabilités, auxquelles Pascal a largement contribué, il est possible de s'intéresser à la notion de hasard et aux statistiques. Expérience intéressante car on peut découvrir que l'indétermination qui paraît liée au hasard peut fréquemment être expliquée (sauf dans certaines situations où les déterminismes sont trop nombreux pour être cernés comme dans le loto). "Ce que nous appelons hasard n'est et ne peut être que la cause ignorée d'un effet connu."

gnant. Parité pour définir la posture de l'enseignant. Asymétrique car l'enseignant pour adopter une posture d'horizontalité vis-à-vis de l'élève ne doit pas occulter la position de verticalité qu'il occupe.

5 Les 16 premiers chiffres de l'écriture décimale de $\pi$ sont 3,141 592653589 793. En 2007, on connait déjà plus de 1012 décimales de $\pi$. 
écrivit Voltaire. Les probabilités nous confrontent au fortuit, à l'imprévisible parfois explicables.

En revenant sur les exemples précédents, on peut convenir que des interrogations métaphysiques aussi puissantes que celles d'infini, d'idéal de la pensée, de réel, de hasard et leurs corollaires, l'ordre, la comparaison, la mesure, l'espace, le temps sont sous-jacents à un enseignement des mathématiques dès l'école primaire. Alors, enseigner des mathématiques, c'est plus que chercher à calculer, à démêler des équations, à démontrer, que même résoudre des problèmes. C'est s'intéresser à ces questionnements qui viennent de la nuit des temps. Et lorsque la résolution de problèmes lui donne corps, c'est retrouver une démarche temporelle car existe de toujours et pour toujours.

\section{Dans les sciences de la vie et de la terre}

Le vivant par son émergence, son évolution et son terme constitue la question fondamentale que chacun se pose pour tenter de répondre à des interrogations comme "qu'est-ce qu'un être vivant? Y a t-il une nature humaine qui nous rapproche et nous distingue des autres êtres vivants?"...

Biologiquement on répond à ces questions en éclairant les notions de phylogenèse (l'histoire évolutive d'une espèce ou d'un groupe d'espèces apparentées de sorte à mieux comprendre leur évolution et à établir une classification de ces espèces en fonction de leur parenté), l'ontogenèse (qui décrit le développement progressif d'un organisme de sa conception à son terme), l'autopoïèse (le pouvoir d'un organisme à maintenir sa structure dans un environnement variable) et la reproduction (l'ensemble des processus par lesquels une espèce se perpétue, en suscitant de nouveaux individus).

Chemin faisant, trois notions émergent : "l'individu", "l'organisme" et le "soi".

Depuis Aristote, la notion d'individu est pensée sur le modèle de l'organisme (ce qui existe dans l'enveloppe charnelle qui s'offre à notre vision), comme si la biologie constituait le domaine privilégié pour concevoir les notions d'individualité et d'identité. Comme si le soi psychologique (pour Carl Jung le mot Soi distingue une personne au-delà de ce qu'elle en perçoit, cette perception étant le Moi) se confondait avec le soi biologique.

La notion d'organisme qui structure toutes les sciences du vivant interroge ces super-organismes que constituent les colonies d'insectes sociaux, en 
particulier, dont il est possible de se demander s'ils doivent être considérés comme des organismes.

A propos du soi, l'immunologie illustre ce paradoxe : un individu qui a subi une greffe (ce qui au niveau psychologique va dans le sens de son maintien en vie et de son espoir de survie) développe des mécanismes de rejet de cette greffe (ce qui au plan biologique illustre la reconnaissance inconsciente de ce qui lui est étranger, et qui est contraire à son aspiration psychologique). Comme si le soi biologique était ignorant du soi psychologique.

Ces trois notions d'individu, d'organisme, de soi font émerger des questionnements ontologiques comme celui "du même et de l'autre ou de l'un et du multiple" (la physiologie propose une explication unique du fonctionnement des organismes; seule la médecine illustre le différent sous le commun), comme celui du "suis-je mon corps ou autre chose que mon corps?" (qui émerge lorsque je ne parviens pas physiquement à faire ce que je souhaite faire et que se pose la question de la relation entre le corps et la pensée au sens restreint, entre la matière et la pensée au sens large), comme celui du moment où le mot vie est justifié (avec les questions du début et de la fin de la vie; le foetus est-il un être humain, la vie existe t-elle après un électro-encéphalogramme plat?), de l'unité dans la diversité (un éléphant, un homme et un microbe sont trois êtres vivants alors qu'extérieurement il existe peu de points communs).

L'écologie est une branche de la biologie dans les programmes enseignés sous le vocable des sciences de la vie et de la terre. Les études concernant les écosystèmes, et plus largement la biosphère sont elles aussi l'objet de réflexions philosophiques. Des problèmes émergent à la frontière entre l'écologie et la société, et l'apparition de nouvelles interrogations éthiques en constituent un développement naturel qui réfère à la diversité biologique aux pratiques du développement durable, aux répercussions variées des OGM sur les systèmes écologiques, sociologiques et économiques.

Un enseignement des sciences de la vie et de la terre apparait ainsi comme un embrayeur de questionnements éthiques et psychologiques, comme une occasion de dépasser des visions réductionnistes ou vitalistes et de déboucher sur des questions ontologiques comme celle qu'illustra Jacques Monod avec son "Le hasard et la nécessité" (MONOD, 1970), que Francisco Varéla (1988) poursuivit avec la notion d'émergence. Questions toujours sans réponse définitive qui illustrent bien l'idée de questionnements fondamentaux disciplinaires: lorsque des questions internes à une discipline qui ont des réponses au sein de cette discipline amènent à se poser des questions plus larges, philosophiques au sens large, ontologiques et métaphysiques. 


\section{Conclusion provisoire}

Pierre Thuilier dans le modèle qu'il propose quant à l'évolution des disciplines suggère indirectement que les $\mathrm{D}$. des disciplines, ne proposant pas de nouveaux concepts sont parvenues à une phase académique correspondant à la stabilisation du cadre de recherche, en attente d'une révolution scientifique qui vienne reconsidérer ce paradigme.

Je pense avoir montré que la $\mathrm{D}$. des disciplines peut se reconstruire autour d'une centration sur les concepts de sens, de rapport au savoir ou de culture très proches car nimbés d'une dimension anthropologique.

Le rapport au savoir constitue une théorie de référence pour analyser le fonctionnement scolaire dans sa totalité. Car si nous avons parlé de rapport au savoir de l'élève, il faudrait utiliser de cette notion pour tous les acteurs de l'école, enseignants, personnels d'encadrement, parents et tutelle. Du reste la question de rapport au savoir, plus qu'une théorie de référence est un véritable paradigme, à savoir un critère d'intelligibilité de la chose scolaire.

Le sens, que l'on retrouve en philosophie et en psychanalyse constitue une des questions clés que pose tout objet, toute situation, toute personne à celui qui cherche à le comprendre, à lui trouver une signification. Deux grandes tendances alors se font jour. Les fidèles de la logique réduisent la signification à un "vrai/ faux" ou à un "dénué de sens/doué de sens". Les partisans de l'interprétation adoptent une liberté de juger, qui loin de réduire le sens à deux pôles, font référence à la polysémie, à un infini d'explications possibles.

Si le rapport au savoir constitue un paradigme qui permet d'analyser et de comprendre les comportements scolaires des acteurs de cette institution, le sens est au delà et en deçà de ce paradigme. Le sens qu'un élève trouve ou non à l'école en tant qu'institution de dépliement des savoirs est fonction en grande partie de son rapport au savoir. Le sens en est la conséquence et la cause.

La culture, à travers la dimension ontologique que recèlent tous les contenus de savoirs est ce vers quoi tend l'école: faire découvrir à l'élève que les disciplines et les savoirs qu'elles renferment sont autant de façon d'approcher de grandes interrogations philosophiques à dimension universelle.

Ainsi nous suggérons que les $\mathrm{D}$. des disciplines - attentives aux questions d'apprentissage, d'enseignement, de formation - dans un champ de savoirs et de pratiques à propos d'une matière à transmettre, ont des fondements épistémologiques (ceux auxquelles les D. des disciplines se sont jusqu'alors intéressées) mais tout autant ontologiques. 
Comment dès lors définir la $\mathrm{D}$. des disciplines? Comme une approche épistémologico-ontologico-anthropologique des questions de transmission de contenus et de pratiques pour une discipline donnée.

\section{RÉFÉRENCES}

ARTIGUE, M.; DOUADY, R. La didactique des mathématiques en France-Emergence d'un champ scientifique. Disponible au Persée: $<$ http://www.persee.fr/web/revues/home/ prescript/article/rfp_0556-7807_1986_num_76_1_1503 >. Accès le: 22 fév. 2015.

MONOD J. Le hasard et la nécessité, Essai sur la philosophie naturelle de la biologie moderne. Paris: éditions du Seuil, 1970.

VARÉLA, F. Autonomie et connaissance, essai sur le vivant. Paris: éditions du Seuil, Seuil, 1988.

\section{OUVRAGES PUBLIÉS D’AUTEUR}

DEVELAY, M. Comment refonder l'école primaire? Regards croisés, Bruxelles: De Boeck, 2013.316 p.

DEVELAY, M. D'un programme de connaissances à un curriculum de compétences. De Boeck, En cours d'édition (sortie début 2015).

DEVELAY, M. De l'apprentissage à l'enseignement. 6è édit. Paris: E.S.F, 1992. 167 p. DEVELAY, M. Donner du sens à l'école. 4è édit. Paris: E.S.F, 1996. 124 p.

DEVELAY, M. Parents, comment éduquer votre enfant. Paris: E.S.F, 1998. 124 p.

DEVELAY, M. Peut-on former les enseignants? 3è édit. Paris: E.S.F, 1994. 156 p.

DEVELAY, M. Propos sur les sciences de l'éducation, réflexions épistémologiques. 2è édit. Paris: ESF, 2001. 124 p.

DEVELAY, M. Savoirs scolaires et didactiques des disciplines. Une encyclopédie pour aujourd'hui. Paris: E.S.F, 1995. 355 p.

DEVELAY, M.; ASTOLFI, J. P. La didactique des sciences. 6è édit. Paris: PUF, Que sais-je, 1989. 124 p.

DEVELAY, M.; LÉVINE, J. Pour une anthropologie des savoirs scolaires. Paris: ESF, 2003. 124 p. 
DEVELAY, M.; MEIRIEU, P. Émile, reviens vite. Ils sont devenus fous. 3è édit. Paris: E.S.F, 1992. 211 p.

DEVELAY, M.; MEIRIEU, P. Le transfert de connaissances en formation initiale et continue. Actes de colloque, CRDP Lyon, 1996.

DEVELAY, M. et al. Quelle école voulons-nous? Dialogue sur l'école avec la Ligue de l'enseignement. Paris: ESF, 2001. 188 p.

Texto recebido em 04 de outubro de 2015. Texto aprovado em 11 de outubro de 2015. 
